\title{
Small Intestine Obstruction in Patients due to Phytobezoar: Case Report of Four Patients
}

\author{
${ }^{1}$ VR Anil Kumar, ${ }^{2}$ Anupama K Pujar, ${ }^{3}$ Ashwini Kumar Kudari, ${ }^{4}$ Kush K Luthra
}

\begin{abstract}
Introduction: Bezoars are rare cause of small-bowel obstruction and lead to intraluminal obstruction. The other causes are foreign bodies, gall stones, and enteroliths. Phytobezoars are collection of non-digestible materials, usually of vegetable origin, and are most commonly found in patients with impaired gastric emptying, on high-fiber diet and with abnormal food habits.

We present a case report of four patients who presented with acute intestinal obstruction, and the etiology in all these four cases was found to be a phytobezoar. The complaints of diffuse pain abdomen, vomiting, and constipation were common in all four cases. One patient had undergone a gastric pull-up surgery for carcinoma esophagus and one female had undergone bilroth1 for peptic ulcer disease. One patient was a known case of diabetes mellitus and had undergone surgery for perforated peptic ulcer few years back.
\end{abstract}

Conclusion: Diagnosis of bezoars is usually confirmed by barium examination or endoscopy, and definitive treatment is surgery in case of obstruction with the removal of bezoar.

Keywords: Intestinal obstruction, Intraluminal mass, Phytobezoar.

How to cite this article: Kumar VRA, Pujar AK, Kudari AK, Luthra KK. Small Intestine Obstruction in Patients due to Phytobezoar: Case Report of Four Patients. Int J Recent Surg Med Sci 2016;2(1):40-43.

\section{Source of support: Nil}

Conflict of interest: None

\section{INTRODUCTION}

Phytobezoars are the most frequent type of bezoars and occur from ingestion of non-digestible fibers. These usually occur in two forms, either take the form of orange pith or pulp in patients who have undergone surgery in past or persimmon in patients without previous surgery. ${ }^{1}$ High concentration of tannin, a monomer that polymerizes in the presence of gastric acid, is present in

\footnotetext{
${ }^{1,3,4}$ Associate Professor, ${ }^{2}$ Resident

${ }^{1}$ Department of Emergency Medicine, M.S. Ramaiah Medical College, Bengaluru, Karnataka, India

${ }^{2-4}$ Department of General Surgery, M.S. Ramaiah Medical College, Bengaluru, Karnataka, India
}

Corresponding Author: Kush K Luthra, Associate Professor Department of General Surgery, M.S. Ramaiah Medical College Bengaluru, Karnataka, India, Phone: +919611377613, e-mail: kush.luthra01@gmail.com persimmon. The tannin undergoes polymerization and then acts as a nucleus for bezoar formation. Normally, vegetable fibers that cannot pass through the pylorus undergo hydrolysis within the stomach. After softening they easily go through the small bowel. The gastric motility becomes slightly abnormal and delayed in patients who have undergone any gastric surgery in the past. Owing to decreased gastric acidity and rapid emptying of stomach, there is an increased possibility of bezoar formation. Primary small-bowel bezoars are very rare and normally forms in patients with an underlying smallbowel disease like diverticulum, stricture, or tumor. Phytobezoar can also develop secondarily in patients with strictures caused by Crohn's disease, tuberculosis, previous surgeries, or with small-bowel diverticula. In such cases, bile constituents or calcium salts contribute to bezoar development. ${ }^{2}$ We present a case report of small intestinal obstruction caused by phytobezoar.

In this case report, swallowed foreign bodies may have been involved, although a foreign body that has passed the pylorus is usually able to pass through the remainder of the small bowel without difficulty, unless the small bowel is already compromised by postoperative adhesions. Anatomically, the narrowest part of the small bowel is the terminal ileum, and the peristalsis is weaker here in comparison to the more proximal segments. The intramural width of the small bowel is measured by taking plain abdominal radiographs of a gas-filled lumen, and an intramural width of $3 \mathrm{~cm}$ is abnormal and is suggestive of obstruction or ileus. Certain radiologic investigations are in routine practice to confirm the diagnosis and severity of a small-bowel obstruction, but not the etiology. ${ }^{3}$ The investigation of choice in patients with suspected smallbowel obstructions is an abdominal X-ray, and this method should always be performed first. ${ }^{3}$ A bowel larger than $3 \mathrm{~cm}$ in diameter is diagnostic of obstruction. Gas or fluid is usually present in the distended small bowel loops or they have both gas and fluid within them; hence the presence of air-fluid levels on an abdominal X-ray film at different levels is always suggestive of intestinal obstruction. ${ }^{3}$

\section{CASE REPORTS}

\section{Case 1}

A 27-year-old male presented to the emergency department with history of distention of abdomen, vomiting, 
and constipation since 2 days. The patient had colicky pain of sudden onset and non-projectile bilious vomiting. The patient had a good built and consumed a high-fiber diet with lots of cereals and there was no history of any previous gastric surgery or any intestinal pathology. His vital signs are blood pressure at 130/78 $\mathrm{mm} \mathrm{Hg}$ and a heart rate of 120 beats/minute on admission. On examination of the abdomen it was found that there was distension of abdomen with diffuse generalized tenderness and absence of bowel sounds, and rectal examination revealed an empty rectum with no palpable mass. The electrocardiogram was within normal limits. A clinical diagnosis of intestinal obstruction was then made based on the radiological findings (Fig. 1). An exploratory laparotomy was subsequently performed on the patient and resection anastomosis was done. Operative findings were of dilated small bowel loops proximal to the obstruction and phytobezoar causing obstruction at mid ileum that was confirmed by ileostomy. The jejunum and ileum were dilated and hypertrophied, but no jejunal or ileal mass or polyps were found (Fig. 2). The postoperative

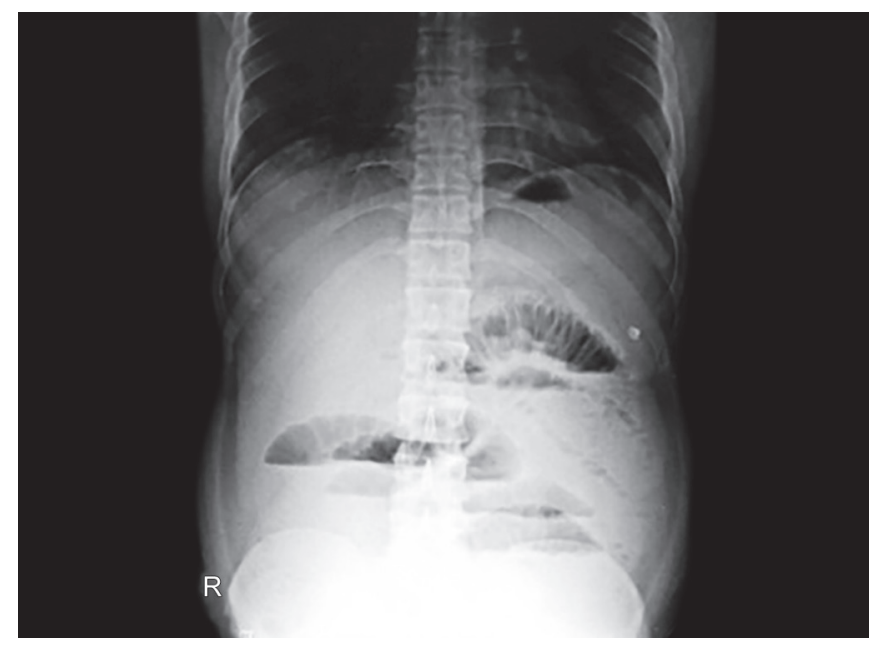

Fig. 1: X-ray erect abdomen: Showing air fluid levels suggestive of small bowel obstruction

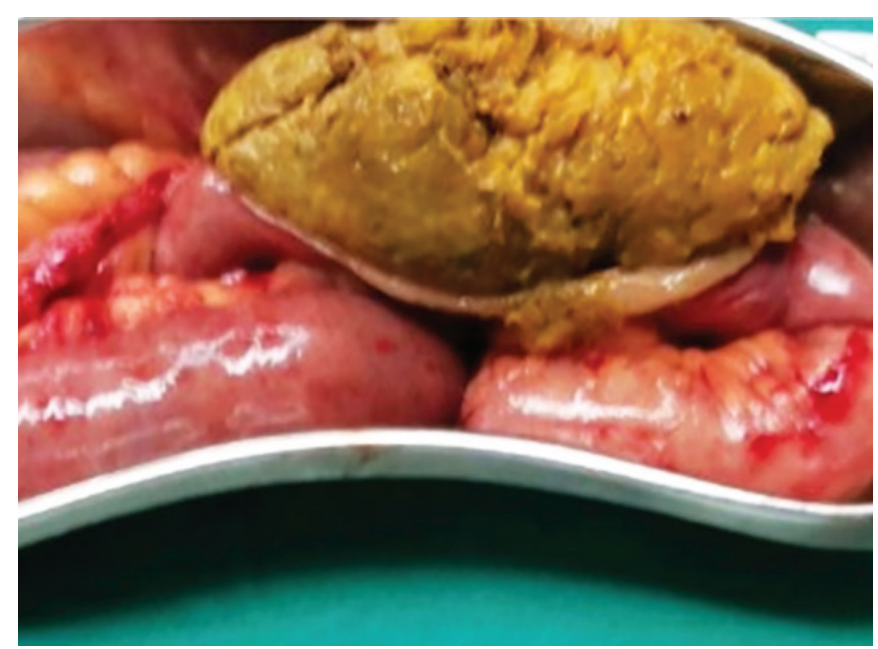

Fig. 3: Postoperative image showing phytobezoar period was uneventful (Fig. 3), during which the patient was started on nourishing fluid, antibiotics, and a soft diet. He was discharged 4 days later. The histopathology report of the operative specimen and its contents confirmed the degenerate vegetable matter.

\section{Case 2}

A 57-year-old male patient was brought to casualty with complaints of diffuse pain and with distension of abdomen since 1 day. He had four episodes of vomiting and was not passing flatus and stool. Past history of patient revealed that he had undergone laparotomy for peptic ulcer perforation 4 years back. On examination it was found that the abdomen was distended with diffuse tenderness. X-ray of abdomen standing showed air-fluid levels and dilated bowel loops (Fig. 4). The diagnosis was confirmed as acute intestinal obstruction due to adhesions. Operative findings were of dilated loops from ileocaecal junction to mid jejunum. Faecolith was found at the distal ileum and enterotomy was done. Histopathological confirmation of faecolith showed vegetative material. The

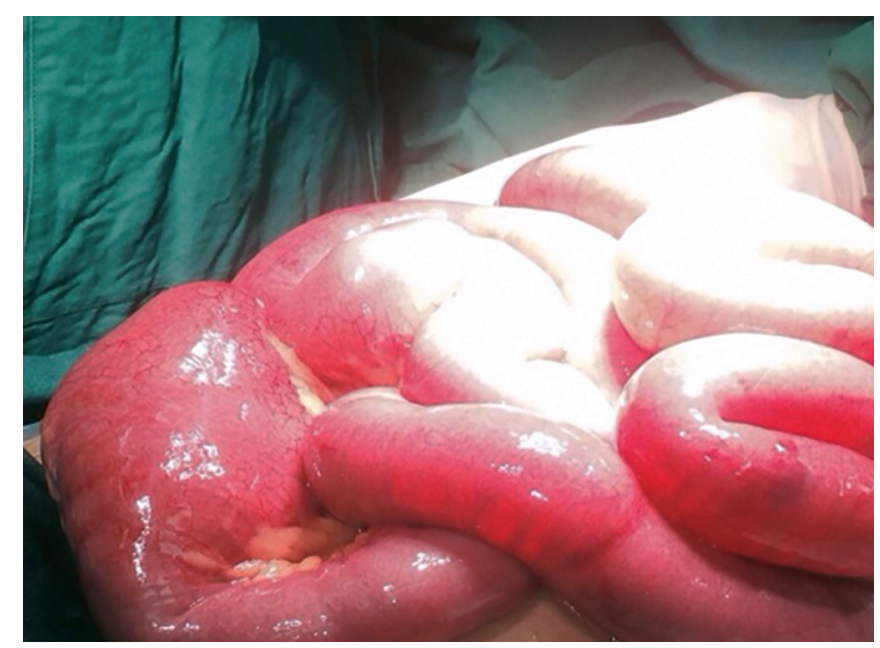

Fig. 2: Intraoperative picture showing site of obstruction

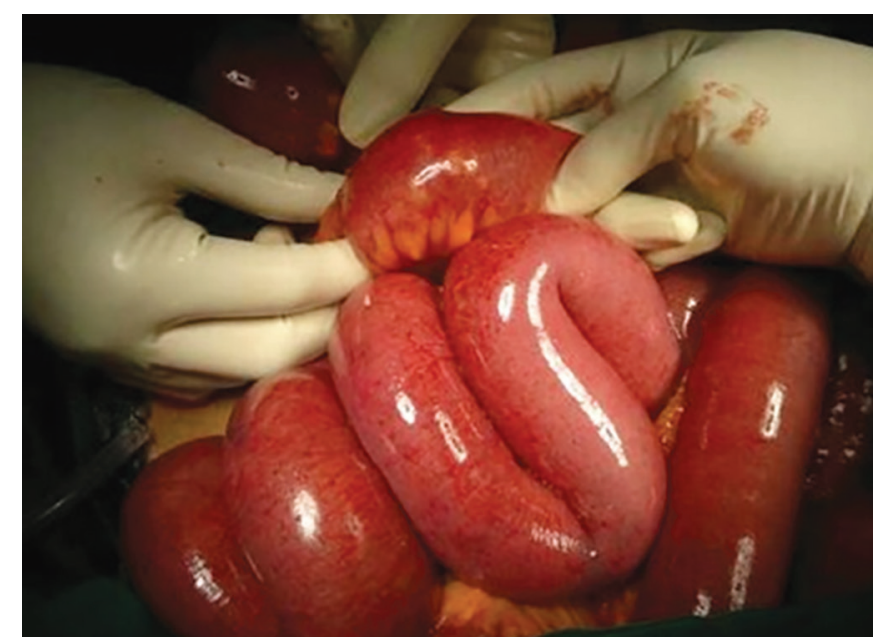

Fig. 4: Dilated small bowel loops with intraluminal mass being felt 
patient was kept nil per oral for 4 days and clear liquids were allowed on 5 th day followed by soft diet. The patient responded well to treatment and was discharged in stable condition on prokinetics.

\section{Case 3}

A 68-year-old diabetic patient presented with classical signs of intestinal obstruction since 3 days. Past history revealed that he had undergone surgery for carcinoma esophagus. He had gastric paresis. Diagnosis of obstruction was confirmed by X-ray of abdomen standing. The patient was managed conservatively and kept nil per oral with Ryle's tube insertion. The patient did not respond to conservative management and was posted for surgery. Operative findings showed dilated small-bowel loops with impacted mass at distal ileum. Histopathology confirmed the diagnosis of phytobezoar. Patient was discharged on 13th day in stable condition.

\section{Case 4}

The 4th case in our case report is of a 70-year-old female who presented with classical signs of intestinal obstruction since 4 days. The patient had undergone bilrothh1 gastrectomy 20 years back for peptic ulcer disease. She also had complaints of excessive bloating and on and off constipation since 1 month. Diagnosis was confirmed by X-ray abdomen standing and USG abdomen. During surgery an impacted faecolith was found in distal ileum. Enterotomy was done to remove it (Fig. 5), and on histopathology the material was found to be vegetative (Fig. 6). The patient was discharged in stable condition on 10th day.

\section{DISCUSSION}

Small-bowel obstructions account for $20 \%$ of hospital admissions. The most common cause is adhesions in a previously operated patient, and other causes include

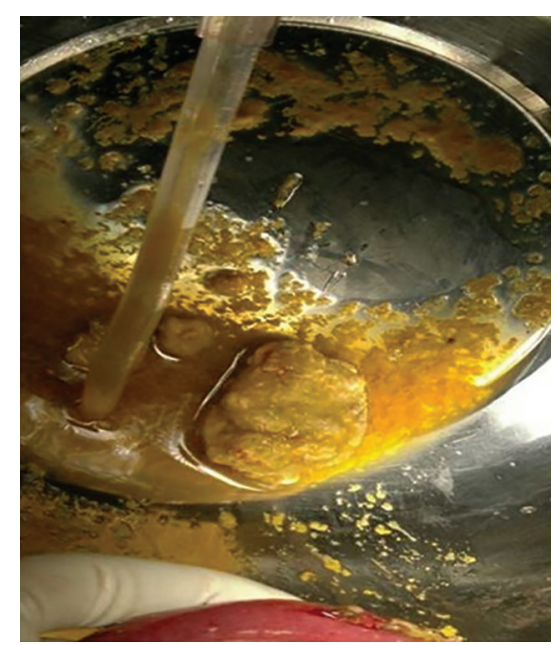

Fig. 5: Phytobezoar being removed after enterotomy malignancies of the bowel, strangulated and obstructed hernia, and inflammatory bowel disease. Phytobezoars are a very rare cause and accounts for only 0.4 to $4 \%$ cases. There is no particular age or sex prevalence observed for occurrence of phytobezoars. ${ }^{4}$ There are four types of bezoars: Phytobezoars, trichobezoars, pharmacobezoars, and lactobezoars. Phytobezoars are the most common among all types of bezoars, and are composed of vegetable matter (celery, pumpkin, grape skin, prune, and persimmons) and contain a large amount of nondigestible fibers (cellulose, hemicellulose, lignin, and fruit tannins). In trichobezoars there is gastric concretion of hair fibers and is usually seen in patients with a history of psychiatric predisposition and in children with mental retardation. Pharmacobezoars, an another type of bezoar, consist of medications, such as cholestyramine, kayexalate resin, cavafate, and antacids, which adhere when taken in bulk. Lastly, lactobezoars are seen in low birth weight neonates who are fed on highly concentrated formula within their first week of life. ${ }^{5}$

Clinically, the primary small-bowel bezoars always present as intestinal obstructions and usually become impacted in the narrowest portion of the small bowel, the most common site being the terminal ileum, as was found in our patients, followed by the jejunum. ${ }^{6}$ More than half of reported cases of patients with phytobezoars had a history of gastric surgery. ${ }^{7}$ But in our report there was only one patient with past history of gastric surgery and the other with gastric pull-up procedure. A plain abdominal radiograph typically shows a classical air-fluid levels suggestive of obstruction. Occasionally, we may be able to see the outline of a bezoar, which is actually difficult to differentiate from abscess or feces within the colon. Ultrasound has been used to detect bezoar. In a retrospective study done by Ripolles et al, ${ }^{8}$ ultrasound was able to detect phytobezoar in $88 \%$ of patients with small-bowel obstructions, but in our study ultrasound was not helpful

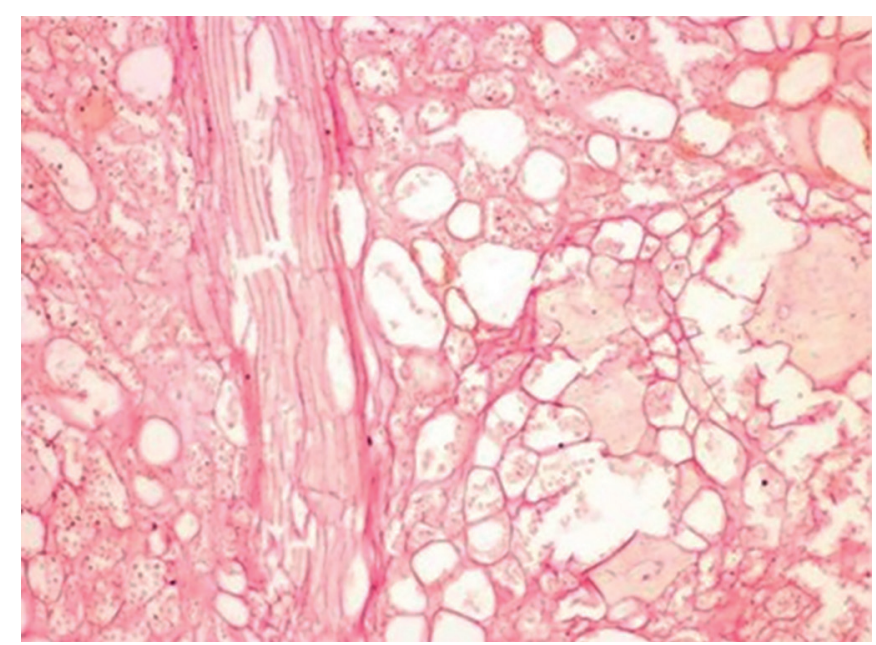

Fig. 6: Histopathology showing vegetable matter 
in detecting bezoars as a cause of obstruction. A bezoar appears as a hyperechoic arc-like surface with acoustic shadowing on ultrasound; however, this feature may cause difficulty in differentiating bezoar from gallstones, which have similar ultrasound characteristics.

\section{CONCLUSION}

Once the diagnosis has been made, treatment may consist of medical management, endoscopic removal, or surgery. Dissolution therapy with papain, cellulose, or even coca cola has been described, albeit with variable results. ${ }^{9,10}$ Most bezoars require endoscopic therapy to fragment the bezoar and remove the pieces ${ }^{11}$ with high success rates. Surgery is required if endoscopic treatment fails or if complications, such as bleeding, obstruction, or perforation occur. It is mandatory to explore the whole gastrointestinal tract in order to avoid synchronous bezoar and the recurrence of intestinal obstruction due to a retained bezoar. Prevention includes avoidance of high-fiber foods, introduction of prophylactic medication to improve gastric emptying, and psychological or psychiatric follow-up in patients with psychiatric disease. ${ }^{4}$ In difficult, recurrent cases, periodic endoscopy with repeated mechanical disruption is necessary.

\section{REFERENCES}

1. Acar T, Tuncal S, Aydin R. An unusual cause of gastrointestinal obstruction: Bezoar. N Z Med J 2003 May;116(1173):U422.
2. Kim JH, Ha HK, Sohn MJ, Kim AY, Kim TK, Kim PN, Lee MG, Myung SJ, Yang SK, Jung HY, Kim JH. CT findings of phytobezoar associated with small bowel obstruction. Eur Radiol 2003 Feb;13(2):299-304.

3. DiSantis DJ, Ralls PW, Balfe DM, Bree RL, Glick SN, Levine MS, Megibow AJ, Saini S, Shuman WP, Greene FL, et al. The patient with suspected small bowel obstruction: Imaging strategies. American College of Radiology. ACR Appropriateness Criteria. Radiology 2000 Jun; (Suppl 215): 121-124.

4. Kalogeropoulou C, Kraniotis P, Zabakis P. Small bowel obstruction due to phytobezoar: CT findings. Eur Assoc Radiol 2003; Clinical case 2840.

5. Andrus $\mathrm{CH}$, Ponsky JL. Bezoars: classification, pathophysiology and treatment. Am J Gastroenterol 1988 May;83(5): 476-478.

6. Teo M, Wong $\mathrm{CH}$, Chui CH, Chow P, Soo KC. Food bolus - An uncommon cause of small intestinal obstruction. Aust N Z J Surg 2003;73 (Suppl 1):A47.

7. Lee JF, Leow CK, Lai PB, Lau WY. Food bolus intestinal obstruction in a Chinese population. Aust N Z J Surg 1997 Dec;67(12):866-868.

8. Rippolés T, Garcia-Aguayo J, Martinez MJ, Gil P. Gastrointestinal bezoars: sonographic and CT characteristics. AJR 2001 Jul;177(1):65-69.

9. Lee B-J, Park J-J, Chun H-J, Kim JH, Yeon JE, Jeen YT, Kim JS, Byun KS, Lee SW, Choi JH, et al. How good is cola for dissolution of gastric phytobezoars? World J Gastroenterol 2009 May;15(18):2265.

10. Walker-Renard P. Update on the medical management of phytobezoars. Am J Gastroentrol 1993 Oct;88:1663.

11. Wang YG, Seitz U, Li ZL, Soehendra N, Qiao XA. Endoscopic management of huge bezoars. Endoscopy 1998 May;30(4):371. 